\title{
APPROXIMATION METHODS IN THE THEORY OF HYBRID DIFFERENTIAL EQUATIONS WITH LINEAR PERTURBATIONS OF SECOND TYPE
}

\author{
BAPURAO C. DHAGE
}

\begin{abstract}
In this paper, some existence theorems for the extremal solutions are proved for an initial value problem of nonlinear hybrid differential equations via constructive methods. The monotone iterative techniques for initial value problems of first order hybrid differential equations are developed and it is shown that the sequences of successive iterations defined in a certain way converge to the minimal and maximal solutions of the hybrid differential equations.
\end{abstract}

\section{Introduction}

It is well known that the Banach contraction mapping principle is the only fixed point theorem in nonlinear analysis which provides a useful method for approximating a unique solution for the initial and boundary value problems of ordinary differential equations via successive iterations. However, to the best of our knowledge, there is no such fixed point theorem or method developed so far for the hybrid differential equations without further assumptions on the nonlinearities involved in the equations. Recently, Dhage and Jadhav [6] and Dhage and Lakshmikantham [5] have proved some basic results for the hybrid differentials equations of first order with the linear and quadratic perturbations of second type. In this paper, using the ideas from Lakshmikantham and Leela [8], Dhage [4] and Ladde et al. [7], we establish some theoretical approximation results for the extremal solutions of hybrid differential equations between the given lower and upper solutions.

Given a closed and bounded interval $J=\left[t_{0}, t_{0}+a\right]$ in $\mathbb{R}, \mathbb{R}$ the real line, for some $t_{0} \in \mathbb{R}$, $a \in \mathbb{R}$ with $a>0$, consider an initial value problem of first order ordinary hybrid differential equations (in short HDE),

$$
\left\{\begin{aligned}
\frac{d}{d t}[x(t)-f(t, x(t))] & =g(t, x(t)), t \in J, \\
x\left(t_{0}\right) & =x_{0} \in \mathbb{R},
\end{aligned}\right.
$$

Received December 16, 2012, accepted March 9, 2013.

2010 Mathematics Subject Classification. 34A12, 34A45.

Key words and phrases. Hybrid differential equation, existence theorem, Upper and lower solutions, monotone iterative technique. 
where, $f, g: J \times \mathbb{R} \rightarrow \mathbb{R}$ are continuous.

By a solution of the HDE (1.1) we mean a function $x \in C(J, \mathbb{R})$ such that

(i) the function $t \mapsto x-f(t, x)$ is continuous for each $x \in \mathbb{R}$, and

(ii) $x$ satisfies the equations in (1.1).

where $C(J, \mathbb{R})$ is the space of continuous real-valued functions defined on $J$.

The HDE (1.1) is a linear perturbation of second type of an initial value problem of first order nonlinear differential equations and has been discussed in Dhage and Jadhav [6] for existence theory for different aspects of the solutions. The details of different types of nonlinear perturbations of a differential equation appears in Dhage [3]. The specialty of the results of present paper lies in our constructive approach for the solutions to the HDE (1.1) on $J$.

The following hypotheses concerning the function $f$ is sometime crucial in the study of HDE (1.1).

$\left(\mathrm{A}_{0}\right)$ The function $x \mapsto x-f\left(t_{0}, x\right)$ is injective in $\mathbb{R}$, and

$\left(\mathrm{B}_{0}\right)$ The function $g$ is bounded real-valued function on $J \times \mathbb{R}$.

Note that hypothesis $\left(\mathrm{A}_{0}\right)$ holds in particular if the function $x \mapsto x-f\left(t_{0}, x\right)$ is increasing in $\mathbb{R}$. Again, hypothesis $\left(\mathrm{B}_{0}\right)$ is much common and widely used in the literature in the study of nonlinear differential equations.

We shall also make use of the following result in what follows.

Lemma 1.1. Assume that hypothesis $\left(\mathrm{A}_{0}\right)-\left(\mathrm{B}_{0}\right)$ hold. Then a function $x$ is a solution of the HDE (1.1) if and only if it is a solution of the hybrid integral equation (HIE),

$$
x(t)=\left[x_{0}-f\left(t_{0}, x_{0}\right)\right]+f(t, x(t))+\int_{t_{0}}^{t} g(s, x(s)) d s, t \in J .
$$

Proof. Assume first that $x$ is a solution of the HDE (1.1) defined on $J$. Then, by definition of the mapping, $t \mapsto x(t)-f(t, x(t))$ is continuous, and so, is differentiable, whence $\frac{d}{d t}[x(t)-f(t, x(t))]$ is Reimann integrable on $J$. Applying integration to (1.1) from $t_{0}$ to $t$, we obtain the HIE (1.2) on $J$.

Conversely, assume that the function $x$ satisfies the HIE (1.2) on $J$. Since $g(t, x)$ is bounded, it can be proved that the function $t \mapsto x(t)-f(t, x(t))$ is continuous for each $x \in C\left(J, \mathbb{R}_{+}\right)$and hence almost everywhere differential on $J$. By a direct differentiation of the HIE (1.2), we obtain the HDE (1.1). Again, substituting $t=t_{0}$ in the HIE (1.1) yields

$$
x\left(t_{0}\right)-f\left(t_{0}, x\left(t_{0}\right)\right)=x_{0}-f\left(t_{0}, x_{0}\right) .
$$


Since the mapping $x \mapsto x-f(t, x)$ is injective in $\mathbb{R}$, we obtain $x\left(t_{0}\right)=x_{0}$. Hence the proof of the lemma is complete.

In the following section, we prove an existence result for the HDE (1.1) in a closed set formed by the lower and upper solutions under some suitable conditions via a hybrid fixed point theorem due to Dhage [1,2].

\section{Method of lower and upper solutions}

In this section we prove an existence result for the HDE (1.1) in a closed and bounded subset given by lower and upper solutions. A construction result is also obtained at the end of the section.

Definition 2.1. A function $u \in C(J, \mathbb{R})$ is said to be a lower solution for the HDE (1.1) defined on $J$ if

(i) $t \mapsto u(t)-f(t, u(t))$ is continuous, and

(ii) $\frac{d}{d t}[u(t)-f(t, u(t))] \leq g(t, u(t)), t \in J, u\left(t_{0}\right) \leq x_{0}$.

Similarly, a function $v \in C(J, \mathbb{R})$ is said to be a upper solution for the HDE (1.1) defined on $J$ if

(i) $t \mapsto v(t)-f(t, v(t))$ is continuous, and

(ii) $\frac{d}{d t}[v(t)-f(t, v(t))] \geq g(t, v(t)), t \in J, v\left(t_{0}\right) \geq x_{0}$.

A solution of the HDE (1.1) is a lower as well an upper solution and vice versa.

If we know the existence of lower and upper solutions of the HDE (1.1) such that $u(t) \leq$ $v(t), t \in J$, then we can prove the existence of a solution of the HDE (1.1) in the closed set

$$
\bar{\Omega}=\{x \in E: u(t) \leq x \leq v(t), t \in J\}
$$

We place the problem under study in the space $C(J, \mathbb{R})$ of continuous real-valued functions defined on $J$. Clearly, $C(J, \mathbb{R})$ is a Banach space with respect to the usual supremum norm $\|\cdot\|$ defined by

$$
\|x\|=\sup _{t \in J}|x(t)| .
$$

We consider the following hypotheses in what follows.

$\left(\mathrm{A}_{1}\right)$ The function $x \mapsto x-f(t, x)$ is increasing in $\mathbb{R}$ for all $t \in J$. 
$\left(\mathrm{A}_{2}\right)$ There exists a constant $L>0$ such that

$$
|f(t, x)-f(t, y)| \leq \frac{L|x-y|}{M+|x-y|}
$$

for all $t \in J$ and $x, y \in \mathbb{R}$. Moreover, $L \leq M$.

$\left(\mathrm{B}_{1}\right)$ There exists a constant $K>0$ such that

$$
|g(t, x)| \leq K
$$

for all $t \in J$ and for all $x \in \mathbb{R}$.

The following existence result is proved in Dhage and Jadhav [6] via a fixed point technique formulated in Dhage [2].

Theorem 2.1. Assume that hypotheses $\left(A_{1}\right)-\left(A_{2}\right)$ and $\left(B_{1}\right)$ hold. Then the HDE (1.1) has a solution on $\mathrm{J}$.

Theorem 2.2. Let $u, v \in C(J, \mathbb{R})$ be lower and upper solutions of $H D E(1.1)$ satisfying $u(t) \leq v(t)$, $t \in J$ and let the hypotheses $\left(A_{1}\right)-\left(A_{2}\right)$ and $\left(B_{1}\right)$ hold. Then, there exists a solution $x(t)$ of $(1.1)$ in the closed set $\bar{\Omega}$, that is, $u(t) \leq x(t) \leq v(t), t \in J$.

Proof. Define a function $p: J \times \mathbb{R} \rightarrow \mathbb{R}$ by

$$
p(t, x)=\max \{u(t), \min \{x(t), v(t)\}\}
$$

Then $\tilde{g}(t, x)=g(t, p(t, x))$ defines a continuous extension of $g$ on $J \times \mathbb{R}$ satisfying

$$
|\tilde{g}(t, x)|=|g(t, p(t, x))| \leq K, t \in J
$$

for all $x \in \mathbb{R}$. Hence by Theorem 2.1, the HDE

$$
\left\{\begin{aligned}
\frac{d}{d t}[x(t)-f(t, x(t))] & =\tilde{g}(t, x(t)), t \in J, \\
x\left(t_{0}\right) & =x_{0} \in \mathbb{R}
\end{aligned}\right.
$$

has a solution $x$ defined on $J$.

For any $\epsilon>0$, define

$$
u_{\epsilon}(t)-f\left(t, u_{\epsilon}(t)\right)=(u(t)-f(t, u(t)))-\epsilon(1+t)
$$

and

$$
v_{\epsilon}(t)-f\left(t, v_{\epsilon}(t)\right)=(v(t)-f(t, v(t)))+\epsilon(1+t)
$$


for $t \in J$. Then in view of hypotheses $\left(\mathrm{A}_{1}\right)$, we obtain

$$
u_{\epsilon}(t)<u(t) \text { and } \quad v(t)<v_{\epsilon}(t)
$$

for $t \in J$.

Since

$$
u\left(t_{0}\right) \leq x_{0} \leq v\left(t_{0}\right)
$$

one has

$$
u_{\epsilon}\left(t_{0}\right)<x_{0}<v_{\epsilon}\left(t_{0}\right)
$$

Next, we shall show that

$$
u_{\epsilon}(t)<x(t)<v_{\epsilon}(t), t \in J
$$

Define $X(t)=x(t)-f(t, x(t)), t \in J$. Similarly, define

$$
U_{\epsilon}(t)=u_{\epsilon}(t)-f\left(t, u_{\epsilon}(t)\right), \quad U(t)=u(t)-f(t, u(t)),
$$

and

$$
V_{\epsilon}(t)=v_{\epsilon}(t)-f\left(t, v_{\epsilon}(t)\right), \quad V(t)=v(t)-f(t, v(t))
$$

for all $t \in J$.

If (2.7) is not true, then there exists a $t_{1} \in\left(t_{0}, t_{0}+a\right]$ such that

$$
x\left(t_{1}\right)=v_{\epsilon}\left(t_{1}\right)
$$

and

$$
u_{\epsilon}(t)<x(t)<v_{\epsilon}(t), t_{0} \leq t<t_{1} .
$$

If $x\left(t_{1}\right)>v\left(t_{1}\right)$, then $p\left(t_{1}, x\left(t_{1}\right)\right)=v\left(t_{1}\right)$. Moreover,

$$
u\left(t_{1}\right) \leq p\left(t_{1}, x\left(t_{1}\right)\right) \leq v\left(t_{1}\right)
$$

Now,

$$
V^{\prime}\left(t_{1}\right) \geq g\left(t_{1}, v\left(t_{1}\right)\right)=\tilde{g}\left(t_{1}, x\left(t_{1}\right)\right)=X^{\prime}(t)
$$

for all $t \in J$. Since

$$
V_{\epsilon}(t)>V^{\prime}(t)
$$

for all $t \in J$, we have that

$$
V_{\epsilon}^{\prime}\left(t_{1}\right)>X^{\prime}\left(t_{1}\right)
$$

However,

$$
X\left(t_{1}\right)=V_{\epsilon}\left(t_{1}\right)
$$


and

$$
X(t)=V_{\epsilon}(t), t_{0} \leq t<t_{1}
$$

together imply that

$$
\frac{X\left(t_{1}+h\right)-X\left(t_{1}\right)}{h}>\frac{V_{\epsilon}\left(t_{1}+h\right)-V_{\epsilon}\left(t_{1}\right)}{h}
$$

if $h<0$ small. Taking the limit as $h \rightarrow 0$ in the above inequality yields

$$
X^{\prime}\left(t_{1}\right) \geq V_{\epsilon}^{\prime}\left(t_{1}\right)
$$

which is a contradiction to (2.8). Hence,

$$
x(t)<v_{\epsilon}(t)
$$

for all $t \in J$. Consequently,

$$
u_{\epsilon}(t)<x(t)<v_{\epsilon}(t), t \in J
$$

Letting $\epsilon \rightarrow 0$ in the above inequality, we obtain

$$
u(t) \leq x(t) \leq v(t), t \in J
$$

This completes the proof.

The existence of lower and upper solutions is an essential ingredient in many problems of nonlinear differential equations and which do exist for every differential equation obviously. The following simple result gives the sufficient conditions that guarantee the existence of lower and upper solutions for the HDE (1.1) defined on $J$.

We consider the following hypothesis:

$\left(\mathrm{B}_{2}\right)$ The function $x \mapsto g(t, x)$ is nonincreasing in $\mathbb{R}$ for all $t \in J$.

Theorem 2.3. Suppose that hypotheses $\left(A_{1}\right)-\left(A_{2}\right)$ and $\left(B_{1}\right)-\left(B_{2}\right)$ hold. Then there exists a lower $u_{0}$ and an upper solutions $v_{0}$ for the HDE (1.1) such that $u_{0}(t) \leq v_{0}(t)$ on $J$.

Proof. Let $y(t)$ be the unique solution of the HDE

$$
\left\{\begin{aligned}
\frac{d}{d t}[y(t)-f(t, y(t))] & =\tilde{g}(t, 0), \quad t \in J, \\
y\left(t_{0}\right) & =x_{0}, \in \mathbb{R} .
\end{aligned}\right.
$$

Define

$$
u_{0}(t)-f\left(t, u_{0}(t)\right)=y(t)-f(t, y(t))-R_{0}, t \in J
$$


and

$$
v_{0}(t)-f\left(t, v_{0}(t)\right)=y(t)-f(t, y(t))+R_{0}, t \in J
$$

for some real number $R_{0}>0$. Choose $R_{0}$ so large that

$$
u_{0}(t)-f\left(t, u_{0}(t)\right) \leq 0 \leq v_{0}(t)-f\left(t, v_{0}(t)\right)
$$

Since $g(t, x)$ is nonincreasing in $x$, one has

$$
u_{0}(t)-f\left(t, u_{0}(t)\right) \leq y(t)-f(t, y(t)) \leq g\left(t, u_{0}(t)\right), t \in J
$$

and

$$
v_{0}(t)-f\left(t, v_{0}(t)\right) \geq y(t)-f(t, y(t)) \geq g\left(t, u_{0}(t)\right), t \in J
$$

Also from (2.10) and (2.11), it follows that

$$
u_{0}\left(t_{0}\right) \leq x_{0} \leq v_{0}\left(t_{0}\right)
$$

Thus, the functions $u_{0}(t)$ and $v_{0}(t)$ are respectively the desired lower and upper solutions for the HDE (1.1) on $J$. Finally, from the hypothesis $\left(A_{1}\right)$ and the inequality (2.12) it follows that $u_{0}(t) \leq v_{0}(t)$ and the proof of the theorem is complete.

Remark 2.1. Assume that all the hypotheses of Theorem 2.2 hold with $\bar{\Omega}$ is replaced with

$$
\bar{\Omega}=\left\{x \in \mathbb{R} \mid u_{0}(t) \leq x \leq v_{0}(t), t \in J\right\} .
$$

Then, by Theorem 2.1, there exists a solution for the HDE (1.1) in the vector segment $\left[u_{0}, v_{0}\right]$ in the Banach space $C(J, \mathbb{R})$. The uniqueness of $x(t)$ is a consequence of nonincreasing nature of $g(t, x)$ in $x$ for each $t \in J$.

\section{Monotone iterative technique}

In this section, we describe a constructive method that yields monotone sequences which converge to the extremal solutions of the HDE (1.1) on $J$. This method is known as monotone iterative technique in the theory of nonlinear analysis and has been employed by several authors for a number of nonlinear differential equations in the literature. This method generates the sequences of successive iterations where the first iteration is a solution of a certain linear differential equation which can be computed explicitly. The advantage of monotone iterative technique lies in the fact that it gives some qualitative information about the solutions of nonlinear differential equation in question and the disadvantage is that unlike Picard's iterations, it does not give any information about the degree of approximation of the solutions, 
that is, how far away the successive iterations are from the actual solution of the prolem in question. The details of monotone iterative technique and applications appears in a monograph by Ladde et al. [7]. Below we apply the monotone iterative technique to HDE (1.1) under some suitable conditions for proving the existence of extremal solutions.

We need the following hypotheses in what follows.

$\left(\mathrm{B}_{3}\right)$ There exists a real number $M>0$ such that

$$
g(t, x)-g(t, y) \geq-M[(x-f(t, x))-(y-f(t, y))]
$$

for each $t \in J$ and $x, y \in \mathbb{R}$ with $x \geq y$.

$\left(\mathrm{B}_{4}\right)$ There exists a constant $K>0$ such that

$$
|\tilde{g}(t, x)|=|g(t, x)+M(x-f(t, x))| \leq K
$$

for all $t \in J$ and $x \in \mathbb{R}$.

Theorem 3.1. Let $u_{0}$ and $v_{0}$ be respectively the lower and upper solutions for the HDE (1.1) satisfying $u_{0}(t) \leq v_{0}(t)$ on $J$ and let hypotheses $\left(A_{1}\right)-\left(A_{2}\right)$ and $\left(B_{3}\right)-\left(B_{4}\right)$ hold. Then there exist monotone sequences $\left\{u_{n}\right\}$ and $\left\{v_{n}\right\}$ such that $u_{n} \rightarrow u$ and $v_{n} \rightarrow v$ uniformly on $J$, where $u$ and $v$ are respectively the minimal and maximal solutions of the HDE (1.1) on J and

$$
u_{0} \leq u_{1} \leq \cdots \leq u_{n} \leq v_{n} \leq \cdots \leq v_{2} \leq v_{1} \leq v_{0}
$$

Proof. For any $\eta \in C(J, \mathbb{R})$ with $u_{0}(t) \leq \eta(t) \leq v_{0}(t)$ on $J$, consider a hybrid differential equation,

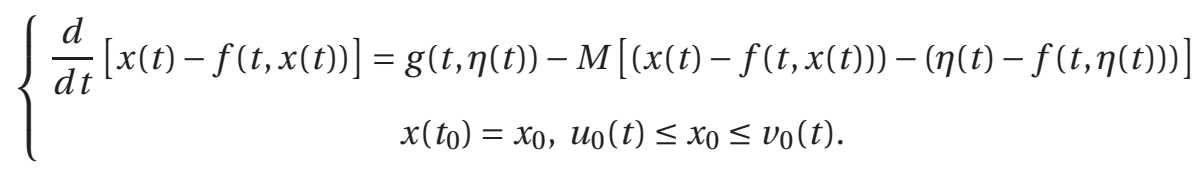

for all $t \in J$. Now the HDE (3.2) is equivalent to the problem

$$
\begin{gathered}
\frac{d}{d t}[x(t)-f(t, x(t))]+M[x(t)-f(t, x(t))]=\tilde{g}(t, \eta(t)), t \in J, \\
x\left(t_{0}\right)=x_{0} .
\end{gathered}
$$

Using the integration factor, the above equation can be put in the form

$$
\begin{aligned}
\frac{d}{d t}\left[e^{M t}(x(t)-f(t, x(t)))\right] & =e^{M t} \tilde{g}(t, \eta(t)), t \in J, \\
x\left(t_{0}\right) & =x_{0} .
\end{aligned}
$$


By lemma 1.1, the above hybrid differential equation is equivalent to he HIE

$$
x(t)=f(t, x(t))+e^{-M\left(t-t_{0}\right)}\left(x_{0}-f\left(t_{0}, x_{0}\right)\right)+\int_{t_{0}}^{t} e^{-M(t-s)} \tilde{g}(s, \eta(s)) d s, t \in J .
$$

Since hypothesis $\left(\mathrm{B}_{4}\right)$ hold, it is clear that for every $\eta$, there exists a unique solution $x \in$ $C(J, \mathbb{R})$ of the HDE (1.1) defined on $J$ in view of Banach contraction mapping principle.

Define a mapping $A$ on $\left[u_{0}, v_{0}\right]$ by $A \eta=x$. This mapping will be used to define sequences $\left\{u_{n}\right\}$ and $\left\{v_{n}\right\}$. Let us now prove that

(a) $u_{0} \leq A u_{0}$ and $v_{0} \geq A v_{0}$.

(b) $A$ is monotone operator on the sector

$$
\left[u_{0}, v_{0}\right]=\left\{x \in C(J, \mathbb{R}) \mid u_{0}(t) \leq x(t) \leq v_{0}(t), t \in J\right\}
$$

To prove (a), set $A u_{0}=u_{1}$, where $u_{1}$ is a unique solution of the HDE (3.2) on $J$ with $\eta=u_{0}$. Denote

$$
p(t)-f(t, p(t))=\left(u_{1}(t)-f\left(t, u_{1}(t)\right)\right)-\left(u_{0}(t)-f\left(t, u_{0}(t)\right)\right)
$$

for $p \in C(J, \mathbb{R})$. Then $p\left(t_{0}\right)-f\left(t_{0}, p\left(t_{0}\right)\right) \geq 0$, and

$$
\begin{aligned}
\frac{d}{d t}[p(t)-f(t, p(t))] & =\frac{d}{d t}\left[\left(u_{1}(t)-f\left(t, u_{1}(t)\right)\right)\right]-\frac{d}{d t}\left[\left(u_{0}(t)-f\left(t, u_{0}(t)\right)\right)\right] \\
& \geq g\left(t, u_{0}(t)\right)-M\left[\left(u_{1}(t)-f\left(t, u_{1}(t)\right)\right)-\left(u_{0}(t)-f\left(t, u_{0}(t)\right)\right)\right]-g\left(t, u_{0}(t)\right) \\
& =-M[p(t)-f(t, p(t))] .
\end{aligned}
$$

This shows that $p(t)-f(t, p(t)) \geq\left[p\left(t_{0}\right)-f\left(t_{0}, p\left(t_{0}\right)\right)\right] e^{-M t} \geq 0$ for all $t \in J$ and hence from (3.4), we obtain

$$
u_{1}(t)-f\left(t, u_{1}(t)\right) \geq u_{0}(t)-f\left(t, u_{0}(t)\right)
$$

for all $t \in J$. Since hypothesis $\left(\mathrm{A}_{1}\right)$ holds, $u_{0}(t) \leq u_{1}(t)$ for all $t \in J$, or, equivalently, $u_{0} \leq A u_{0}$. In a similar way, we can prove that $v_{0} \geq A v_{0}$.

To prove (b), let $\eta_{1}, \eta_{2} \in\left[u_{0}, v_{0}\right]$ be such that $\eta_{1} \leq \eta_{2}$ on $J$. Then one has

$$
g\left(t, \eta_{1}(t)\right)-g\left(t, \eta_{2}(t)\right) \geq-M\left[\left(\eta_{1}(t)-f\left(t, \eta_{1}(t)\right)\right)-\left(\eta_{2}(t)-f\left(t, \eta_{2}(t)\right)\right)\right]
$$

for all $t \in J$.

Suppose that $x_{1}=A \eta_{1}$ and $x_{2}=A \eta_{2}$ and set

$$
p(t)-f(t, p(t))=\left(x_{2}(t)-f\left(t, x_{2}(t)\right)\right)-\left(x_{1}(t)-f\left(t, x_{1}(t)\right)\right)
$$

for some $p \in C(J, \mathbb{R})$. Then, $p\left(t_{0}\right)-f\left(t_{0}, p\left(t_{0}\right)\right) \geq 0$, and

$$
\frac{d}{d t}[p(t)-f(t, p(t))]=\frac{d}{d t}\left[x_{2}(t)-f\left(t, x_{2}(t)\right)\right]-\frac{d}{d t}\left[x_{1}(t)-f\left(t, x_{1}(t)\right)\right]
$$




$$
\begin{aligned}
= & g\left(t, \eta_{2}(t)\right)-M\left[\left(x_{2}(t)-f\left(t, x_{2}(t)\right)\right)-\left(\eta_{2}(t)-f\left(t, \eta_{2}(t)\right)\right)\right] \\
& -g\left(t, \eta_{1}(t)\right)+M\left[\left(x_{1}(t)-f\left(t, x_{1}(t)\right)\right)-\left(\eta_{1}(t)-f\left(t, \eta_{1}(t)\right)\right)\right] \\
\geq & -M\left[\left(\eta_{2}(t)-f\left(t, \eta_{2}(t)\right)\right)-\left(\eta_{1}(t)-f\left(t, \eta_{1}(t)\right)\right)\right] \\
& -M\left[\left(\eta_{2}(t)-f\left(t, \eta_{2}(t)\right)\right)-\left(\eta_{1}(t)-f\left(t, \eta_{1}(t)\right)\right)\right] \\
& +M\left[\left(x_{1}(t)-f\left(t, x_{1}(t)\right)\right)-\left(\eta_{1}(t)-f\left(t, \eta_{1}(t)\right)\right)\right] \\
= & -M[p(t)-f(t, p(t))] .
\end{aligned}
$$

As before, the foregoing inequality implies that $x_{2} \geq x_{1}$ on $J$ which in turn implies that $A \eta_{2} \geq A \eta_{1}$, proving (b).

Now, we define two sequences $\left\{u_{n}\right\}$ and $\left\{v_{n}\right\}$ by

$$
u_{n}=A u_{n-1} \quad \text { and } \quad v_{n}=A v_{n-1}
$$

for $n=1,2, \ldots$.

From the monotonicity of the operator $A$ it follows that

$$
u_{0} \leq u_{1} \leq u_{2} \leq \cdots \leq u_{n} \leq v_{n} \leq \cdots \leq v_{2} \leq v_{1} \leq v_{0}
$$

It is easy to show that the sequences $\left\{u_{n}\right\}$ and $\left\{v_{n}\right\}$ are uniformly bounded and equicontinuous on $J$. The sequences being monotone, they converge by Arzela-Ascoli theorem, uniformly and monotonically on $J$ to $u$ and $v$ respectively. Obviously, $u$ and $v$ are the solutions of

$$
\left\{\begin{array}{c}
\frac{d}{d t}\left[u_{n}(t)-f\left(t, u_{n}(t)\right)\right]=g\left(t, u_{n-1}(t)\right)-M\left[\left(u_{n}(t)-f\left(t, u_{n}(t)\right)\right)\right. \\
\left.-\left(u_{n-1}(t)-f\left(t, u_{n-1}(t)\right)\right)\right] \\
u_{n}\left(t_{0}\right)=x_{0},
\end{array}\right.
$$

and

$$
\left\{\begin{array}{c}
\frac{d}{d t}\left[v_{n}(t)-f\left(t, v_{n}(t)\right)\right]= \\
g\left(t, v_{n-1}(t)\right)-M\left[\left(v_{n}(t)-f\left(t, v_{n}(t)\right)\right)\right. \\
\left.-\left(v_{n-1}(t)-f\left(t, v_{n-1}(t)\right)\right)\right] \\
v_{n}\left(t_{0}\right)=x_{0} .
\end{array}\right.
$$

To prove that $u$ and $v$ are extremal solutions of the HDE (1.1) on $J$, we have to show that if $x$ is any other solution of the HDE (1.1) such that $u_{0}\left(t_{0}\right) \leq x_{0} \leq v_{0}\left(t_{0}\right), t \in J$, then

$$
u_{0}(t) \leq u(t) \leq x(t) \leq v(t) \leq v_{0}(t), t \in J
$$

Suppose that for some $n \in \mathbb{N}, u_{n} \leq x \leq v_{n}$ on $J$ and set

$$
p(t)-f(t, p(t))=(x(t)-f(t, x(t)))-\left(u_{n+1}(t)-f\left(t, u_{n+1}(t)\right)\right)
$$


for some $p \in C(J, \mathbb{R})$. Then, $p\left(t_{0}\right)-f\left(t_{0}, p\left(t_{0}\right)\right)=0$, and

$$
\begin{aligned}
\frac{d}{d t}[p(t)-f(t, p(t))]= & \frac{d}{d t}[x(t)-f(t, x(t))]-\frac{d}{d t}\left[u_{n+1}(t)-f\left(t, u_{n+1}(t)\right)\right] \\
= & g(t, x(t))-g\left(t, u_{n}(t)\right)-M\left[\left(u_{n+1}(t)-f\left(t, u_{n+1}(t)\right)\right)\right. \\
& \left.-\left(u_{n}(t)-f\left(t, u_{n}(t)\right)\right)\right] \\
\geq & -M\left[(x(t)-f(t, x(t)))-\left(u_{n}(t)-f\left(t, u_{n}(t)\right)\right)\right] \\
& +M\left[\left(u_{n+1}(t)-f\left(t, u_{n+1}(t)\right)\right)-\left(u_{n}(t)-f\left(t, u_{n}(t)\right)\right)\right] \\
= & -M[p(t)-f(t, p(t))] .
\end{aligned}
$$

This implies as before that $u_{n+1}(t) \leq x(t)$ for all $t \in J$. Similarly, it is proved that $x(t) \leq$ $v_{n+1}(t)$ for all $t \in J$. Since $u_{0} \leq x \leq v_{0}$ on $J$, we have, by induction principle that $u_{n} \leq x \leq v_{n}$ on $J$ for each $n, n=0,1,2, \ldots$. Taking the limit as $n \rightarrow \infty$, we conclude that $u \leq x \leq v$ on $J$. Hence $u$ and $v$ are respectively the minimal and maximal solutions for the HDE (1.1) on $J$. This completes the proof.

To state a special case of Theorem 3.1, we need the following hypothesis in what follows.

$\left(\mathrm{B}_{5}\right)$ The function $x \mapsto g(t, x)$ is nondecreasing in $\mathbb{R}$ for each $t \in J$.

Corollary 3.2. Let $u_{0}$ and $v_{0}$ be respectively the lower and upper solutions for the HDE (1.1) on $J$ satisfying $u_{0}(t) \leq v_{0}(t)$ on $J$ and let all the conditions of Theorem 3.1 are satisfied with hypothesis $\left(B_{4}\right)$ replaced with $\left(B_{5}\right)$. Then the HDE (1.1) has extremal solutions on J.

The proof of Corollary 3.2 follows from Theorem 3.1 by replacing the constant $M$ in hypothesis $\left(\mathrm{B}_{3}\right)$ with $M=0$. Next, we discuss the case when $g(t, x)$ is nonincreasing in $x$ almost everywhere for $t \in J$. Let $u_{0}$ and $v_{0}$ be respectively the lower and upper solutions for the HDE (1.1) on $J$. Then, consider the two sequences $u_{n}$ and $v_{n}$ of iterations defined as follows:

$$
\frac{d}{d t}\left[u_{n+1}(t)-f\left(t, u_{n+1}(t)\right)\right]=g\left(t, u_{n}(t)\right), \quad t \in J, \quad u_{n+1}\left(t_{0}\right)=u_{0} ;
$$

and

$$
\frac{d}{d t}\left[v_{n+1}(t)-f\left(t, v_{n+1}(t)\right)\right]=g\left(t, v_{n}(t)\right), \quad t \in J, \quad v_{n+1}\left(t_{0}\right)=u_{0} ;
$$

for $n=0,1,2, \ldots$.

Below in the following we show that each one of the sequences $u_{n}$ and $v_{n}$ has two alternating sequences which converge uniformly and monotonically to the extremal solutions of the HDE (1.1) on $J$.

Theorem 3.3. Let hypotheses $\left(A_{1}\right)-\left(A_{2}\right)$ and $\left(B_{5}\right)$ hold. Then either, 
(i) the iterates $\left\{u_{n}\right\}$ given by (3.10) and a unique solution $x$ of the HDE (1.1) defined on $J$ satisfy

$$
u_{0} \leq u_{2} \leq \cdots \leq u_{2 n} \leq x(t) \leq u_{2 n+1} \leq \cdots \leq u_{3} \leq u_{1}
$$

for all $t \in J$, provided $u_{0}(t) \leq u_{2}(t), t \in J$. Furthermore, the sequences $\left\{u_{2 n}\right\}$ and $\left\{u_{2 n+1}\right\}$ converge uniformly and monotonically to $u_{*}$ and $u^{*}$ respectively satisfying $u_{*}(t) \leq x(t) \leq$ $u^{*}(t)$ for all $t \in J$; or

(ii) the iterates $\left\{v_{n}\right\}$ given by (3.11) and a unique solution $x$ of the HDE (1.1) defined on $J$ satisfy

$$
v_{1} \leq v_{3} \leq \cdots \leq u_{2 n+1} \leq x(t) \leq v_{2 n} \leq \cdots \leq v_{2} \leq v_{0}
$$

for all $t \in J$, provided $v_{2}(t) \leq v_{0}(t), t \in J$. Furthermore, the sequences $\left\{v_{2 n}\right\}$ and $\left\{v_{2 n+1}\right\}$ converge uniformly and monotonically to $v_{*}$ and $v^{*}$ respectively satisfying $v_{*}(t) \leq x(t) \leq$ $v^{*}(t)$ for all $t \in J$.

In fact, since the extremal solutions are unique, $u_{*}=v_{*}=u$ and $u^{*}=v^{*}=v$ on $\mathrm{J}$ satisfying $u(t) \leq x(t) \leq v(t), t \in J$.

Proof. By Theorem 2.2, there exist a lower solution $u_{0}$, an upper solution $v_{0}$ and a solution $x$ for the HDE (1.1) such that

$$
u_{0}(t) \leq x(t) \leq v_{0}, \quad t \in J
$$

We shall only prove the case (i), since the proof of case (ii) follows with similar arguments. Assume $u_{0} \leq u_{2}$ on $J$. We shall first show that

$$
u_{0}(t) \leq u_{2}(t) \leq x(t) \leq u_{3}(t) \leq u_{1} t, t \in J
$$

Set

$$
p(t)-f(t, p(t))=\left(u_{1}(t)-f\left(t, u_{1}(t)\right)\right)-\left(u_{0}(t)-f\left(t, u_{0}(t)\right)\right)
$$

for $t \in J$. Then,

$$
\begin{aligned}
\frac{d}{d t}[p(t)-f(t, p(t))] & =\frac{d}{d t}\left[u_{1}(t)-f\left(t, u_{1}(t)\right)\right]-\frac{d}{d t}\left[u_{0}(t)-f\left(t, u_{0}(t)\right)\right] \\
& \geq g\left(t_{0}, u_{0}(t)\right)-g\left(t_{0}, u_{0}(t)\right) \\
& =0
\end{aligned}
$$

and $p\left(t_{0}\right)=0$. Hence,

$$
u_{1}(t)-f\left(t, u_{1}(t)\right) \geq u_{0}(t)-f\left(t, u_{0}(t)\right)
$$

for all $t \in J$. This further in view of hypothesis $\left(\mathrm{A}_{1}\right)$ implies that $u_{1}(t) \geq u_{0}(t)$ on $J$. 
Next, let

$$
p(t)-f(t, p(t))=(x(t)-f(t, x(t)))-\left(u_{1}(t)-f\left(t, u_{1}(t)\right)\right)
$$

for $t \in J$. Then,

$$
\begin{aligned}
\frac{d}{d t}[p(t)-f(t, p(t))] & =\frac{d}{d t}\left[u_{1}(t)-f\left(t, u_{1}(t)\right)\right]-\frac{d}{d t}\left[u_{0}(t)-f\left(t, u_{0}(t)\right)\right] \\
& =g(t, x(t))-g\left(t, u_{0}(t)\right) \\
& \leq 0
\end{aligned}
$$

and $p\left(t_{0}\right)=0$. This implies

$$
x(t)-f(t, x(t)) \leq u_{1}(t)-f\left(t, u_{1}(t)\right)
$$

for all $t \in J$. Since hypothesis $\left(\mathrm{A}_{1}\right)$ holds, one has $x(t) \leq u_{1}(t)$ on $J$. By using similar arguments, we can show successively that

$$
u_{2}(t) \leq x(t), \quad u_{3}(t) \leq u_{1}(t), \text { and } x(t) \leq u_{3}(t), \quad t \in J
$$

Consequently, we have proved that (3.14) holds for $t \in J$.

To prove (3.12), we use the induction principle, i.e. assume that (3.12) is true for some $n$ and show that it holds for $(n+1)$. Consider,

$$
p(t)-f(t, p(t))=\left(u_{2 n+2}(t)-f\left(t, u_{2 n+2}(t)\right)\right)-\left(u_{2 n+1}(t)-f\left(t, u_{2 n+1}(t)\right)\right) .
$$

Then, by using the monotone character of $g$, we have

$$
\begin{aligned}
\frac{d}{d t}[p(t)-f(t, p(t))] & =\frac{d}{d t}\left[\left(u_{2 n+2}(t)-f\left(t, u_{2 n+2}(t)\right)\right)\right]-\frac{d}{d t}\left[u_{2 n+1}(t)-f\left(t, u_{2 n+1}(t)\right)\right] \\
& =g\left(t, u_{2 n+2}(t)\right)-g\left(t, u_{2 n+1}(t)\right) \\
& \leq 0
\end{aligned}
$$

and $p(0)=0$. This shows $p(t) \leq 0$ and hence $u_{2 n+2}(t) \leq u_{2 n+1}(t)$. By repeating similar arguments we can get

$$
u_{0} \leq u_{2} \leq \cdots \leq u_{2 n} \leq u_{2 n+2} \leq x \leq u_{2 n+3} \leq u_{2 n+1} \leq \cdots \leq u_{3} \leq u_{1}
$$

on $J$. Since (3.12) is true for $n=1$, it follows by induction principle that (3.12) is true for all $n$. It is easy to conclude that the sequences $\left\{u_{2 n}\right\},\left\{u_{2 n+1}\right\}$ are uniformly bounded and equicontinuous and hence by Arzela-Ascoli theorem, converge uniformly and monotonically to $u_{*}(t), u^{*}(t)$ respectively and that $u_{*}(t) \leq x(t) \leq u^{*}(t)$ on $J$. This proves the assertion (i) and the proof of Theorem 3.3 is complete. 
Corollary 3.4. In addition to the assumptions of Theorem 3.3, suppose that

$$
g\left(t, u_{1}(t)\right)-g\left(t, u_{2}(t)\right) \geq-M\left[\left(u_{1}(t)-f\left(t, u_{1}(t)\right)\right)-\left(u_{2}(t)-f\left(t, u_{2}(t)\right)\right)\right]
$$

for all $t \in J$, wherever $u_{1}(t) \geq u_{2}(t)$ on $J$. Then $u(t)=v(t)=x(t)$ on $J$.

We note that in the proof of Theorem 3.3, $u$ and $v$ are indeed quasi solutions for the HDE (1.1) since we have that

$$
\frac{d}{d t}[u(t)-f(t, u(t))]=g(t, v(t)), t \in J, u\left(t_{0}\right)=x_{0},
$$

and

$$
\frac{d}{d t}[v(t)-f(t, v(t))]=g(t, u(t)), t \in J, v\left(t_{0}\right)=x_{0}
$$

\section{Mixed monotone iterative technique}

From the discussion of the previous section, it is clear that if the nonlinearity $g(t, x)$ in the HDE (1.1) is either nondecreasing or nonincreasing in the state variable $x$, then we can construct the monotone sequences of successive iterations that converge to the extremal solutions between the given lower and upper solutions of the related hybrid differential equation on $J$. Now we treat the case when $g$ is neither nondecreasing nor nonincreasing in the state variable $x$. If it is possible to split the function $g$ into two components as

$$
g(t, x)=g_{1}(t, x)+g_{2}(t, x)
$$

where, one component $g_{1}(t, x)$ is nondecreasing while another component $g_{2}(t, x)$ is nonincreasing in the state variable $x$, then in this situation we can also construct the sequences of iterations that converge to the extremal solutions of the HDE (1.1) on $J$.

Now, consider an initial value problem of HDE,

$$
\left\{\begin{aligned}
\frac{d}{d t}(x(t)-f(t, x(t))) & =g_{1}(t, x(t))+g_{2}(t, x(t)), t \in J, \\
x\left(t_{0}\right) & =x_{0} \in \mathbb{R}
\end{aligned}\right.
$$

where, $f \in C(J \times \mathbb{R}, \mathbb{R})$ and $g_{1}, g_{2} \in \mathscr{C}(J \times \mathbb{R}, \mathbb{R})$.

In the following we develop a mixed monotone iterative technique and prove an approximation result for the HDE (4.1) in closed sets formed by the lower and upper solutions. Below we give different notions of lower and upper solutions for the HDE (4.1) on $J$. 
Definition 4.1. Let the functions $\alpha, \beta \in C(J, \mathbb{R})$ satisfy the condition that the maps $t \mapsto \alpha(t)-$ $f(t, \alpha(t))$ and $t \mapsto \beta(t)-f(t, \beta(t))$ are absolutely continuous on $J$. Then the functions $(\alpha, \beta)$ are said to be

(a) mixed lower and upper solutions of type I for the HDE (4.1) on J, if

$$
\left\{\begin{aligned}
\frac{d}{d t}[\alpha(t)-f(t, \alpha(t))] & \leq g_{1}(t, \alpha(t))+g_{2}(t, \beta(t)), \quad t \in J, \\
\alpha\left(t_{0}\right) & \leq x_{0},
\end{aligned}\right.
$$

and

$$
\left\{\begin{aligned}
\frac{d}{d t}[\beta(t)-f(t, \beta(t))] & \geq g_{1}(t, \beta(t))+g_{2}(t, \alpha(t)), \quad t \in J, \\
\beta\left(t_{0}\right) & \geq x_{0}
\end{aligned}\right.
$$

and

(b) mixed lower and upper solutions of type II for the HDE (4.1) on $J$ if

$$
\left\{\begin{aligned}
\frac{d}{d t}[\alpha(t)-f(t, \alpha(t))] & \leq g_{1}(t, \beta(t))+g_{2}(t, \alpha(t)), \quad t \in J, \\
\alpha\left(t_{0}\right) & \leq x_{0},
\end{aligned}\right.
$$

and

$$
\left\{\begin{aligned}
\frac{d}{d t}[\beta(t)-f(t, \beta(t))] & \geq g_{1}(t, \alpha(t))+g_{2}(t, \beta(t)), \quad t \in J, \\
\beta\left(t_{0}\right) & \geq x_{0} .
\end{aligned}\right.
$$

If the equality sign holds in the relations of (4.2) and (4.3), then the pair of functions $(\alpha, \beta)$ together is called a mixed solution of type I for the HDE (4.1) on J. Similarly, if the equality sign holds in the relations of (4.4) and (4.5), then the pair of functions $(\alpha, \beta)$ together is called a mixed solution of type II for the HDE (4.1) on $J$.

We need the following hypothesis in what follows.

$\left(\mathrm{B}_{6}\right)$ The function $g_{1}(t, x)$ is nondecreasing in $x$ and the function $g_{2}(t, x)$ is nonincreasing in $x$ for each $t \in J$.

(B $\left.\mathrm{B}_{7}\right)$ The functions $\left(\alpha_{0}, \beta_{0}\right)$ are mixed lower and upper solutions of type I for the HDE (4.1) on $J$ with $\alpha_{0} \leq \beta_{0}$.

$\left(\mathrm{B}_{8}\right)$ The pair $\left(\alpha_{0}, \beta_{0}\right)$ are mixed lower and upper solutions of type II for the HDE (4.1) on $J$ with $\alpha_{0} \leq \beta_{0}$.

Theorem 4.1. Assume that the hypotheses $\left(A_{1}\right)-\left(A_{2}\right)$ and $\left(B_{6}\right)-\left(B_{7}\right)$ hold. Then there exist monotone sequences $\left\{\alpha_{n}\right\}$ and $\left\{\beta_{n}\right\}$ such that $\alpha_{n} \rightarrow \alpha$ and $\beta_{n} \rightarrow \beta$ uniformly on $J$, where $(\alpha, \beta)$ are mixed extremal solutions of the type I for the HDE (4.1) on J. 
Proof. Consider the following quadratic HDE,

$$
\left\{\begin{aligned}
\frac{d}{d t}\left[\alpha_{n+1}(t)-f\left(t, \alpha_{n+1}(t)\right)\right] & =g_{1}\left(t, \alpha_{n}(t)\right)+g_{2}\left(t, \beta_{n}(t)\right), t \in J, \\
\alpha_{n+1}\left(t_{0}\right) & =x_{0},
\end{aligned}\right.
$$

and

$$
\left\{\begin{aligned}
\frac{d}{d t}\left[\beta_{n+1}(t)-f\left(t, \beta_{n+1}(t)\right)\right] & =g_{1}\left(t, \beta_{n}(t)\right)+g_{2}\left(t, \alpha_{n}(t)\right), t \in J, \\
\beta_{n+1}\left(t_{0}\right) & =x_{0}
\end{aligned}\right.
$$

for $n \in \mathbb{N}$.

Clearly, the HDEs (4.6) and (4.7) have unique solutions $\alpha_{n+1}$ and $\beta_{n+1}$ on $J$ respectively in view of Banach contraction mapping principle. Now we wish to prove that

$$
\alpha_{0} \leq \alpha_{1} \leq \cdots \leq \alpha_{n} \leq \beta_{n} \cdots \leq \beta_{1} \leq \beta_{0}
$$

on $J$ for $n=0,1,2, \ldots$. Let $n=0$ and set

$$
p(t)-f(t, p(t))=\left(\alpha_{0}(t)-f\left(t, \alpha_{0}(t)\right)\right)-\left(\alpha_{1}(t)-f\left(t, \alpha_{1}(t)\right)\right)
$$

for $t \in J$. Then by monotonicity of $g_{1}$ and $g_{2}$, we obtain

$$
\begin{aligned}
\frac{d}{d t}[p(t)-f(t, p(t))] & =\frac{d}{d t}\left[\alpha_{0}(t)-f\left(t, \alpha_{0}(t)\right)\right]-\frac{d}{d t}\left[\alpha_{1}(t)-f\left(t, \alpha_{1}(t)\right)\right] \\
& \leq g_{1}\left(t_{0}, \alpha_{0}(t)\right)+g_{2}\left(t, \beta_{0}(t)\right)-g_{1}\left(t_{0}, \beta_{0}(t)\right)+g_{2}\left(t, \alpha_{0}(t)\right) \\
& =0
\end{aligned}
$$

for all $t \in J$ and $p\left(t_{0}\right)=0$. This implies that

$$
\alpha_{0}(t)-f\left(t, \alpha_{0}(t)\right) \leq \alpha_{1}(t)-f\left(t, \alpha_{1}(t)\right)
$$

for all $t \in J$. As hypothesis $\left(\mathrm{A}_{1}\right)$ holds, one has $\alpha_{0}(t) \leq \alpha_{1}(t)$ for all $t \in J$. Similarly, it is proved that $\beta_{1} \leq \beta_{0}$ on $J$. Again, setting

$$
p(t)-f(t, p(t))=\left(\alpha_{1}(t)-f\left(t, \alpha_{1}(t)\right)\right)-\left(\beta_{1}(t)-f\left(t, \beta_{1}(t)\right)\right)
$$

for $t \in J$. Then, by monotonicity of $g_{1}$ and $g_{2}$, we obtain

$$
\begin{aligned}
\frac{d}{d t}[p(t)-f(t, p(t))] & =\frac{d}{d t}\left[\alpha_{1}(t)-f\left(t, \alpha_{1}(t)\right)\right]-\frac{d}{d t}\left[\beta_{1}(t)-f\left(t, \beta_{1}(t)\right)\right] \\
& \leq g_{1}\left(t_{0}, \alpha_{0}(t)\right)+g_{2}\left(t, \beta_{0}(t)\right)-g_{1}\left(t_{0}, \beta_{0}(t)\right)-g_{2}\left(t, \alpha_{0}(t)\right) \\
& \leq 0
\end{aligned}
$$


for all $t \in J$ and $p\left(t_{0}\right)=0$. This implies that

$$
\alpha_{1}(t)-f\left(t, \alpha_{1}(t)\right) \leq \beta_{1}(t)-f\left(t, \beta_{1}(t)\right)
$$

for all $t \in J$. As hypothesis $\left(\mathrm{A}_{1}\right)$ holds, one has $\alpha_{1}(t) \leq \beta_{1}(t)$ for all $t \in J$.

Now we assume that for some integer $k \in \mathbb{N}$,

$$
\alpha_{k-1} \leq \alpha_{k} \leq \beta_{k} \leq \beta_{k-1}
$$

on $J$. We shall show that

$$
\alpha_{k} \leq \alpha_{k+1} \leq \beta_{k+1} \leq \beta_{k}
$$

Set

$$
p(t)-f(t, p(t))=\left(\alpha_{k}(t)-f\left(t, \alpha_{k}(t)\right)\right)-\left(\alpha_{k+1}(t)-f\left(t, \alpha_{k+1}(t)\right)\right)
$$

for $t \in J$. Then by monotonicity of $g_{1}$ and $g_{2}$, we obtain

$$
\begin{aligned}
\frac{d}{d t}[p(t)-f(t, p(t))] & =\frac{d}{d t}\left[\alpha_{k}(t)-f\left(t, \alpha_{k}(t)\right)\right]-\frac{d}{d t}\left[\alpha_{k+1}(t)-f\left(t, \alpha_{k+1}(t)\right)\right] \\
& \leq g_{1}\left(t, \alpha_{k-1}(t)\right)+g_{2}\left(t, \beta_{k-1}(t)\right)-g_{1}\left(t, \alpha_{k}(t)\right)-g_{2}\left(t, \beta_{k}(t)\right) \\
& \leq 0
\end{aligned}
$$

for all $t \in J$ and $p\left(t_{0}\right)=0$. This implies that

$$
\alpha_{k}(t)-f\left(t, \alpha_{k}(t)\right) \leq \alpha_{k+1}(t)-f\left(t, \alpha_{k+1}(t)\right)
$$

for all $t \in J$. As hypothesis $\left(\mathrm{A}_{1}\right)$ holds, one has $\alpha_{k}(t) \leq \alpha_{k+1}(t)$ for all $t \in J$. Similarly, it can be proved that $\beta_{k+1}(t) \leq \beta_{k}(t), t \in J$.

Similarly, assume that the inequality

$$
\alpha_{k-1} \leq \alpha_{k} \leq \beta_{k} \leq \beta_{k-1}
$$

holds on $J$. We shall show that

$$
\alpha_{k} \leq \alpha_{k+1} \leq \beta_{k+1} \leq \beta_{k}
$$

on $J$. Set

$$
p(t)-f(t, p(t))=\left(\alpha_{k+1}(t)-f\left(t, \alpha_{k+1}(t)\right)\right)-\left(\beta_{k+1}(t)-f\left(t, \beta_{k+1}(t)\right)\right)
$$

for $t \in J$. Then by monotonicity of $g_{1}$ and $g_{2}$, we obtain

$$
\frac{d}{d t}[p(t)-f(t, p(t))]=\frac{d}{d t}\left[\alpha_{k+1}(t)-f\left(t, \alpha_{k+1}(t)\right)\right]-\frac{d}{d t}\left[\beta_{k+1}(t)-f\left(t, \beta_{k+1}(t)\right)\right]
$$




$$
\begin{aligned}
& \leq g_{1}\left(t, \alpha_{k}(t)\right)+g_{2}\left(t, \beta_{k}(t)\right)-g_{1}\left(t, \beta_{k}(t)\right)-g_{2}\left(t, \alpha_{k}(t)\right) \\
& \leq 0
\end{aligned}
$$

for all $t \in J$ and $p\left(t_{0}\right)=0$. This implies that

$$
\alpha_{k+1}(t)-f\left(t, \alpha_{k+1}(t)\right) \leq \beta_{k+1}(t)-f\left(t, \beta_{k+1}(t)\right)
$$

for all $t \in J$. As hypothesis $\left(\mathrm{A}_{1}\right)$ holds, one has $\alpha_{k+1}(t) \leq \beta_{k+1}(t)$ for all $t \in J$.

Now it is easy to prove that the sequences $\left\{\alpha_{n}\right\}$ and $\left\{\beta_{n}\right\}$ are uniformly bounded and equicontinuous and so, have uniformly convergent subsequences on $J$. Since they are monotone sequences, $\left\{\alpha_{n}\right\}$ and $\left\{\beta_{n}\right\}$ converge uniformly and monotonically to $\alpha$ and $\beta$ on $J$ respectively. Obviously the pair $(\alpha, \beta)$ is a mixed solution of the HDE (4.1) on $J$. Finally, we show that $(\alpha, \beta)$ is a mixed minimal and maximal solution of type I for the HDE (4.1) on $J$. Let $x$ be any solution of the $\operatorname{HDE}(4.1)$ on $J$ such that $\alpha_{0} \leq x(t) \leq \beta(t)$ on $J$. Suppose that for some $k \in \mathbb{N}$, $\alpha_{k}(t) \leq x(t) \leq \beta_{k}(t), t \in J$. We shall show that $\alpha_{k+1}(t) \leq x(t) \leq \beta_{k+1}(t), t \in J$. Setting

$$
p(t)-f(t, p(t))=\left(\alpha_{k+1}(t)-f\left(t, \alpha_{k+1}(t)\right)\right)-(x(t)-f(t, x(t)))
$$

for $t \in J$. Then, by monotonicity of $g_{1}$ and $g_{2}$, we obtain

$$
\begin{aligned}
\frac{d}{d t}[p(t)-f(t, p(t))] & =\frac{d}{d t}\left[\alpha_{k+1}(t)-f\left(t, \alpha_{k+1}(t)\right)\right]-\frac{d}{d t}[x(t)-f(t, x(t))] \\
& \leq g_{1}\left(t, \alpha_{k}(t)\right)+g_{2}\left(t, \beta_{k}(t)\right)-g_{1}(t, x(t))-g_{2}(t, x(t)) \\
& \leq 0
\end{aligned}
$$

for all $t \in J$ and $p\left(t_{0}\right)=0$. This implies that

$$
\alpha_{k+1}(t)-f\left(t, \alpha_{k+1}(t)\right) \leq x(t)-f(t, x(t))
$$

for all $t \in J$. As hypothesis $\left(\mathrm{A}_{1}\right)$ holds, one has $\alpha_{k+1}(t) \leq x(t)$ for all $t \in J$. Similarly, it is shown that $x(t) \leq \beta_{k+1}(t)$ for all $t \in J$. By principle of induction method, $\alpha_{n} \leq x \leq \beta_{n}$ on $J$ for all $n \in \mathbb{N}$. Taking the limit as $n \rightarrow \infty$, we obtain $\alpha \leq x \leq \beta$ on $J$. Thus $(\alpha, \beta)$ are the mixed extremal solutions of type I for the HDE (4.1) on $J$, that is,

and

$$
\frac{d}{d t}[\alpha(t)-f(t, \alpha(t))]=g_{1}(t, \alpha(t))+g_{1}(t, \beta(t)), \quad t \in J, \quad \alpha\left(t_{0}\right)=x_{0}
$$

$$
\frac{d}{d t}[\beta(t)-f(t, \beta(t))]=g_{1}(t, \beta(t))+g_{1}(t, \alpha(t)), t \in J, \quad \beta\left(t_{0}\right)=x_{0} .
$$

This completes the proof. 
Corollary 4.2. If in addition to the assumptions of Theorem 4.1, we suppose that for $u_{1} \geq u_{2}$, $u_{1}, u_{2} \in \bar{\Omega}$, we have

$$
g_{1}\left(t, u_{1}(t)\right)-g_{1}\left(t, u_{2}(t)\right) \leq N_{1}\left[\left(u_{1}(t)-f\left(t, u_{1}(t)\right)\right)-\left(u_{2}(t)-f\left(t, u_{2}(t)\right)\right)\right], N_{1}>0,
$$

and

$$
g_{2}\left(t, u_{1}(t)\right)-g_{2}\left(t, u_{2}(t)\right) \leq N_{2}\left[\left(u_{1}(t)-f\left(t, u_{1}(t)\right)\right)-\left(u_{2}(t)-f\left(t, u_{2}(t)\right)\right)\right], N_{2}>0,
$$

then $\alpha(t)=x(t)=\beta(t)$ on $J$.

Proof. Since $\alpha \leq \beta$ on $J$, it is enough to show that $\beta \leq \alpha$ on $J$. Define a function $p \in C(J, \mathbb{R})$ by

$$
p(t)-f(t, p(t))=(\beta(t)-f(t, \beta(t)))-(\alpha(t)-f(t, \alpha(t))) .
$$

Then, $p\left(t_{0}\right)=0$ and

$$
\begin{aligned}
\frac{d}{d t}[p(t)-f(t, p(t))]= & \frac{d}{d t}[\beta(t)-f(t, \beta(t))]-\frac{d}{d t}[\alpha(t)-f(t, \alpha(t))] \\
= & g_{1}(t, \beta(t))-g_{1}(t, \alpha(t))+g_{2}(t, \alpha(t))-g_{2}(t, \beta(t)) \\
\leq & N_{1}[(\beta(t)-f(t, \beta(t)))-(\alpha(t)-f(t, \alpha(t)))] \\
& +N_{2}[(\alpha(t)-f(t, \alpha(t)))-(\beta(t)-f(t, \beta(t)))] \\
= & \left(N_{1}+N_{2}\right)[p(t)-f(t, p(t))] .
\end{aligned}
$$

This shows that $p(t)-f(t, p(t)) \leq 0$ on $J$, proving thereby that $\beta \leq \alpha$ on $J$. Hence $\alpha=x=\beta$ on $J$, completing the proof.

Remark 4.1. As a consequence of Theorem 4.1, we have several important observations. It is noted that many interesting special cases can be derived from Theorem 4.1, some of which are already studied in the earlier results. Below we list our remarks concerning these observations.

(1) In Theorem 4.1, suppose that $g_{2}(t, x)=0$. Then $\alpha_{0}, \beta_{0}$ are natural lower and upper solutions of (4.1) and with $g(t, x)$ nondecreasing, we get the monotone sequences $\left\{\alpha_{n}\right\},\left\{\beta_{n}\right\}$ converging to minimal and maximal solutions of (4.1) respectively, lying in the sector $\left[\alpha_{0}, \beta_{0}\right]$.

(2) However, if $g_{1}(t, x)$ is not nondecreasing and $g_{2}(t, x)=0$, we can assume that $g_{1}(t, x)+$ $M(x-f(t, x))$ is nondecreasing in $x$ for some $M>0$ and still come to the same conclusion as above, since the HDE

$$
\frac{d}{d t}[x(t)-f(t, x(t))]=\tilde{g}_{1}(t, x)(t), t \in J, x\left(t_{0}\right)=x_{0}
$$


satisfies the conditions of Theorem 3.1.

When $g_{1}(t, x)$ is not nondecreasing in $x$, we consider the HDE

$$
\frac{d}{d t}[x(t)-f(t, x(t))]=\tilde{g}_{1}(t, x(t))-M(x(t)-f(t, x(t))), t \in J, x\left(t_{0}\right)=x_{0},
$$

where $\tilde{g}_{1}(t, x)=g_{1}(t, x)+M(x-f(t, x)), M>0$ is nondecreasing in $x$. Note that HDE (4.9) is same as (4.1) with $g_{2}(t, x)=0$. We see that it can also be seen as (4.1) with $g_{1}(t, x)$ replaced by $\tilde{g}_{1}(t, x)$ and $g_{2}(t, x)$ replaced by $-M(x-f(t, x))$. Hence we get the same conclusions as of Theorem 4.1, since $\tilde{g}_{1}(t, x)$ is nondecreasing in $x$ and $-M(x-f(t, x))$ is nonincreasing in $x$.

(3) If $g_{1}(t, x)=0$ in Theorem 4.1, we obtain the result for nonincreasing $g_{2}(t, x)$ in $x$ and the functions $\alpha_{0}, \beta_{0}$ are mixed lower and upper solutions of the HDE

$$
\frac{d}{d t}[x(t)-f(t, x(t))]=g_{2}(t, x(t)), t \in J, \quad \alpha\left(t_{0}\right)=x_{0}
$$

with nonincreasing $g_{2}(t, x)$ in $x$. In this case, the monotone iterates $\left\{\alpha_{n}\right\},\left\{\beta_{n}\right\}$ converge to $\alpha, \beta$ respectively which satisfy

$$
\frac{d}{d t}[\alpha(t)-f(t, \alpha(t))]=g_{1}(t, \beta(t)), t \in J, \alpha\left(t_{0}\right)=x_{0}
$$

and

$$
\frac{d}{d t}[\beta(t)-f(t, \beta(t))]=g_{2}(t, \alpha(t)), t \in J, \beta\left(t_{0}\right)=x_{0} .
$$

(4) If in (3) above, we suppose that $g_{2}(t, x)$ is not nonincreasing in $x$ and there exists a $N>0$ such that $\tilde{g}_{2}(t, x)=g_{2}(t, x)-N(x-f(t, x))$ is nonincreasing in $x$. Then, we can consider the HDE

$$
\frac{d}{d t}[x(t)-f(t, x(t))]=g_{2}(t, x(t))=\tilde{g}_{2}(t, x(t))+N(x(t)-f(t, x(t))), \quad x\left(t_{0}\right)=x_{0}
$$

which is the same as HDE (4.1) with $g_{1}(t, x)$ replaced by $N(x-f(t, x))$ which is nondecreasing in $x$ and $g_{2}(t, x)$ replaced by $\tilde{g}_{2}(t, x)$ which is nonincreasing in $x$. Hence, the present case, then reduces to Theorem 4.1 and the conclusion of Theorem 4.1 remains valid.

(5) Suppose $g_{1}(t, x)$ is nondecreasing but $g_{2}(t, x)$ is not nonincreasing in $x$. Then, consider the HDE

$$
\frac{d}{d t}[x(t)-f(t, x(t))]=\tilde{g}_{1}(t, x(t))+\tilde{g}_{2}(t, x(t)), t \in J, x\left(t_{0}\right)=x_{0},
$$

where $\tilde{g}_{1}(t, x)=g_{1}(t, x)+N(x-f(t, x)), N>0$ is nondecreasing in $x$ and $\tilde{g}_{2}(t, x)=g_{2}(t, x)-$ $N x-f(t, x), N>0$, is nonincreasing in $x$. This results is same as Theorem 4.1 with $g_{1}(t, x), g_{2}(t, x)$ replaced by $\tilde{g}_{1}(t, x), \tilde{g}_{2}(t, x)$ respectively and the conclusion of Theorem 4.1 holds. Note that $\tilde{g}_{1}(t, x(t))+\tilde{g}_{2}(t, x(t))=g_{1}(t, x(t))+g_{2}(t, x(t))$ and hence, HDE (4.10) is the same as the HDE (4.1). 
(6) If $g_{1}(t, x)$ is not nondecreasing in $x$ but $g_{2}(t, x)$ is nonincreasing in $x$, then consider the HDE

$$
\frac{d}{d t}[x(t)-f(t, x(t))]=\tilde{g}_{1}(t, x(t))+\tilde{g}_{2}(t, x(t)), t \in J, x\left(t_{0}\right)=x_{0},
$$

where $\tilde{g}_{1}(t, x)=g_{1}(t, x)+N(x-f(t, x)), N>0$ is nondecreasing and $\tilde{g}_{2}(t, x)=g_{2}(t, x)-$ $N(x-f(t, x)), M>0$ is nonincreasing in $x$. This results is contained in Theorem 4.1 and so, the conclusion of Theorem 4.1 is valid. Again note that HDE (4.11) is the same as (4.1) since $\tilde{g}_{1}(t, x)+\tilde{g}_{2}(t, x)=g_{1}(t, x)+g_{2}(t, x)$.

(7) If $g_{1}(t, x)$ is not nondecreasing and $g_{2}(t, x)$ is not nonincreasing, then for $M>0, N>$ 0 , such that $\tilde{g}_{1}(t, x)=g_{1}(t, x)+M(x-f(t, x))$ is nondecreasing and $\tilde{g}_{2}(t, x)=g_{2}(t, x)-$ $N(x-f(t, x))$ is nonincreasing, we get the context of Theorem 4.1 with $g_{1}(t, x), g_{2}(t, x)$ replaced by $\tilde{g}_{1}(t, x), \tilde{g}_{2}(t, x)$ respectively and hence the conclusion of Theorem 4.1 remains valid.

Next, we consider the case of the mixed lower and upper solutions of type II for the HDE (4.1) and prove the existence of sequences that converge to the mixed extremal solution. Here, we need not assume the existence of mixed lower and upper solutions, since it can be established with the given assumptions.

Theorem 4.3. Assume that the hypotheses $\left(A_{1}\right)-\left(A_{2}\right)$ and $\left(B_{6}\right)-\left(B_{8}\right)$ hold. Then, for any solution $x(t)$ of (4.1) with $\alpha_{0} \leq x \leq \beta_{0}$ on $J$, we have the iterates $\alpha_{n}, \beta_{n}$ satisfying for $t \in J$,

$$
\left\{\begin{array}{l}
\alpha_{0} \leq \alpha_{2} \leq \cdots \leq \alpha_{2 n} \leq x \leq \alpha_{2 n+1} \leq \cdots \leq \alpha_{3} \leq \alpha_{1}, \\
\beta_{1} \leq \beta_{3} \leq \cdots \leq \beta_{2 n+1} \leq x \leq \beta_{2 n} \leq \cdots \leq \beta_{2} \leq \beta_{0},
\end{array}\right.
$$

provided $\alpha_{0} \leq \alpha_{2}$ and $\beta_{2} \leq \beta_{0}$ on $\mathrm{J}$, where the iterates are given by

$$
\left\{\begin{aligned}
\frac{d}{d t}\left[\alpha_{n+1}(t)-f\left(t, \alpha_{n+1}(t)\right)\right] & =g_{1}\left(t, \beta_{n}(t)\right)+g_{2}\left(t, \alpha_{n}(t)\right), t \in J, \\
\alpha_{n+1}\left(t_{0}\right) & =x_{0},
\end{aligned}\right.
$$

and

$$
\left\{\begin{aligned}
\frac{d}{d t}\left[\beta_{n+1}(t)-f\left(t, \beta_{n+1}(t)\right)\right] & =g_{1}\left(t, \alpha_{n}(t)\right)+g_{2}\left(t, \beta_{n}(t)\right), t \in J, \\
\beta_{n+1}\left(t_{0}\right) & =x_{0}
\end{aligned}\right.
$$

for $n \in \mathbb{N}$. Moreover, the monotone sequences $\left\{\alpha_{2 n}\right\},\left\{\alpha_{2 n+1}\right\},\left\{\beta_{2 n}\right\},\left\{\beta_{2 n+1}\right\}$ converge uniformly to $\alpha, \beta, \alpha^{*}, \beta^{*}$ respectively and they satisfy

$$
\begin{aligned}
& \frac{d}{d t}[\alpha(t)-f(t, \alpha(t))]=g_{1}(t, \beta(t))+g_{2}(t, \alpha(t)), \\
& \frac{d}{d t}[\beta(t)-f(t, \beta(t))]=g_{1}(t, \alpha(t))+g_{2}(t, \beta(t))
\end{aligned}
$$




$$
\begin{aligned}
& \frac{d}{d t}\left[\alpha^{*}(t)-f\left(t, \alpha^{*}(t)\right)\right]=g_{1}\left(t, \beta^{*}(t)\right)+g_{2}\left(t, \alpha^{*}(t)\right), \\
& \frac{d}{d t}\left[\beta^{*}(t)-f\left(t, \beta^{*}(t)\right)\right]=g_{1}\left(t, \alpha^{*}(t)\right)+g_{2}\left(t, \beta^{*}(t)\right)
\end{aligned}
$$

for $t \in J$ and $\alpha \leq x \leq \beta, \alpha^{*} \leq x \leq \beta^{*}, t \in J, \alpha(0)=\beta(0)=\alpha^{*}(0)=\beta^{*}(0)=x_{0}$.

Proof. In view of hypothesis (ii), it is easy to construct the mixed lower and upper solutions for the HDE (4.1) following the method of Theorem 2.2. Hence, we proceed by assuming that such mixed lower and upper solutions $\alpha_{0}, \beta_{0}$ of type II exist. Assume further that $\alpha_{0} \leq \alpha_{2}$ and $\beta_{2} \leq \beta_{0}$, on $J$. We show that

$$
\left\{\begin{array}{l}
\alpha_{0} \leq \alpha_{2} \leq x \leq \alpha_{3} \leq \alpha_{1}, \\
\beta_{1} \leq \beta_{3} \leq x \leq \beta_{2} \leq \beta_{0}
\end{array}\right.
$$

on $J$. Set

$$
p(t)-f(t, p(t))=(x(t)-f(t, x(t)))-\left(\alpha_{1}(t)-f\left(t, \alpha_{1}(t)\right)\right) .
$$

Using the fact that $\alpha_{0} \leq x \leq \beta_{0}$ on $J, x$ being any solution of (4.1) and the monotonic nature of the functions $g_{1}$ and $g_{2}$, we obtain

$$
\begin{aligned}
\frac{d}{d t}[p(t)-f(t, p(t))] & =\frac{d}{d t}[x(t)-f(t, x(t))]-\frac{d}{d t}\left[\alpha_{1}(t)-f\left(t, \alpha_{1}(t)\right)\right] \\
& =g_{1}(t, x(t))+g_{2}(t, x(t))-g_{1}\left(t, \beta_{0}(t)\right)-g_{2}\left(t, \alpha_{0}(t)\right) \\
& \leq 0
\end{aligned}
$$

for all $t \in J$ and $p\left(t_{0}\right)=0$. Hence, we conclude

$$
x(t)-f(t, x(t)) \leq \alpha_{1}(t)-f\left(t, \alpha_{1}(t)\right) \quad \text { or } \quad x(t) \leq \alpha_{1}(t)
$$

for all $t \in J$.

Similarly, it can be shown that $\alpha_{3} \leq \alpha_{1}, \beta_{1} \leq x$ and $\alpha_{2} \leq x$, by considering the differences

$$
\begin{aligned}
& p(t)-f(t, p(t))=\left(\alpha_{3}(t)-f\left(t, \alpha_{3}(t)\right)\right)-\left(\alpha_{1}(t)-f\left(t, \alpha_{1}(t)\right)\right), \\
& p(t)-f(t, p(t))=\left(\beta_{1}(t)-f\left(t, \beta_{1}(t)\right)\right)-(x(t)-f(t, x(t)))
\end{aligned}
$$

and

$$
p(t)-f(t, p(t))=\left(\alpha_{2}(t)-f\left(t, \alpha_{2}(t)\right)\right)-(x(t)-f(t, x(t)))
$$

respectively. In each of these cases, we obtain $\frac{d}{d t}[p(t)-f(t, p(t))] \leq 0$, for all $t \in J$ and the claim (4.15) is established. The rest of the proof is similar to Theorem 3.3 with appropriate modifications. Hence we omit the details.

\section{Acknowledgement}

The author is thankful to Prof. Lakshmikatham and Prof. J. Vasundhara Devi for their kind hospitality during my stay at Vishakhapatnam, India, 2011. 


\section{References}

[1] B. C. Dhage, A nonlinear alternative in Banach algebras with applications to functional differential equations, Nonlinear Studies, 13 (2006), 343-354.

[2] B. C. Dhage, A fixed point theorem in Banach algebras with applications to functional integral equations, Kyungpook Math. J., 44 (2004), 145-155.

[3] B. C. Dhage, Quadratic perturbations of periodic boundary value problems of second order ordinary differential equations, Diff. Equ. \& Appl., 2 (2010), 465-486.

[4] B. C. Dhage, Theoretical approximation methods for hybrid differential equations, Dynamic Systems and Applications, 20 (2011), 455-478.

[5] B. C. Dhage and V. Lakshmikatham, Basic results on hybrid differential equations, Nonlinear Analysis: Hybrid Systems, 4 (2010), 414-424.

[6] B. C. Dhage and Namdev S. Jadhav, Basic results on hybrid differential equations with linear perturbation of second type, Tamkang J. Math., 44 (2) (2013), 171-186.

[7] G. S. Ladde, V. Lakshmikantham and A. S. Vatsala, Monotone Iterative Techniques for Nonlinear Differential Equations, Pitman, New York, 1985.

[8] V. Lakshmikantham and S. Leela, Differential and Integral Inequalities, Academic Press, New York, 1969.

Kasubai, Gurukul Colony, Ahmedpur-413 515, Dist: Latur, Maharashtra, India.

E-mail: bcdhage@yahoo.co.in 